\title{
PREFACE
}

\section{Perseveration in Neurogenic Communication Disorders}

To our knowledge, this is the first collection of articles on the topic of perseveration that has appeared in the literature. There are a growing number of publications on the issue, but heretofore they have been scattered in several books and journals. We owe a debt of gratitude to Nancy Helm-Estabrooks for accepting the notion of a special issue of Seminars in Speech and Language devoted to the topic, for giving us the go ahead, and for allowing us to construct the best possible list of contributors.

The initial article by Christman et al, is devoted to teasing apart the distinct types of repetitive verbal outputs, which, although they all involve reiteration of some form or another, do not fall under most accepted definitions of perseveration, whether it be continuous, stuckin-set, or recurrent. Throughout the history of the study of aphasia, there have been numerous efforts to distinguish between many kinds of postactivated verbal behaviors. ${ }^{1}$

The first descriptions of perseveration were functional in nature and consisted of explanatory accounts of the failure of the normal inhibition of a previous activation. There has been an upsurge in the examination of the neuropharmacological underpinnings of apha$\mathrm{sia}^{2}$ and this, in turn, has led to a more focused understanding of the biochemical basis of perseveration. McNamara and Albert outline how catecholaminergic and cholinergic systems control and regulate the executive cognitive functions of focus, resistance to interference, attentional switching, memorial encoding, and retrieval from memory stores-all of which must be operating normally to prevent perseveration. In addition, they demonstrate how dopaminergic agents can increase inhibitory control processes and how cholinesterase inhibitors and other cholinergic agents can help block verbal intrusions. The neuropharmacology involved in the maintenance of homeostasis is one of the most bewilderingly complex processes of human cognition. The processing is extremely rapid, distributed through a complex of neuroanatomic routes, and well out of the way of volitional intent. When perseveration follows from brain damage that interferes with these systems, the automaticity of processing becomes painfully evident in the unwanted reappearance of recently produced elements.

The fluctuating processes of neuropharmacological agents to maintain proper stasis in the system can be, in extremely interesting ways, paralleled in connectionist models, and accordingly connectionist modeling can address an array of findings from neurophysiology. The article by Gotts and Plaut demonstrates how an earlier model developed by Plaut ${ }^{3}$ for describing perseverative errors in optic aphasia on object naming tasks can be extended to account for the more recent findings of subtle paired-associate short-term learning of words linked by contiguity alone. It also recasts accounts of perseveration as arising from weakened/damaged input systems of one sort or another rather than from abnormal amounts of prime strength or slower decay rates of the prime. Mathematically lowering weight values in the input system's long-term memories is the connectionist's way of "deafferenting" one or another linguistic task domain (reading, naming, writing, repeating, and so on) such that there will be a tendency to reproduce the prior productions from the short-term association system. The

Perseveration in Neurogenic Communication Disorders; Editors in Chief, Nancy Helm-Estabrooks, Sc.D., and Nan Bernstein Ratner, Ed.D.; Guest Editors, Hugh W. Buckingham, Ph.D., and Sarah S. Christman, Ph.D. Seminars in Speech and Language, volume 25, number 4, 2004. ${ }^{1}$ Department of Communication Sciences and Disorders, Louisiana State University, Baton Rouge, Louisiana; ${ }^{2}$ Department of Communication Sciences and Disorders, College of Allied Health, University of Oklahoma Health Sciences Center, Oklahoma City, Oklahoma. Copyright (C) 2004 by Thieme Medical Publishers, Inc., 333 Seventh Avenue, New York, NY 10001, USA. Tel: +1(212) 584-4662. 0734-0478,p;2004,25,04, 291,293,ftx,en;ss100211b. 
weights and decay rates in connectionist systems can intriguingly mirror the biochemical interactions at functional levels of description, thus offering yet another example of the unification or "alignment" of two scientific idioms rather than the reduction of one to another. ${ }^{4}$ Gotts and Plaut focus on a supposed deficit of acetylcholine, as do Bayles and her colleagues.

A significant amount of work has been done recently on the neuropsychology and the neuropharmacology of Alzheimer's disease. The contribution by Bayles et al is a clear example of some of the best work done with this population and serves to illustrate again that work in perseveration unifies many distinct scientific approaches to the phenomena. Bayles and colleagues have clearly demonstrated again that a principal biochemical breakdown in Alzheimer's patients rests with acetylcholine and that perseveration is task sensitive, which in turn supports the recent findings in neuropsychology that perseverative behavior must have a pre-existing weakening in some input domain. The task that taps that domain will be the task that elicits the most perseveration. The powerful statistics used in this contribution serve to carve patient severity and task type along fairly clear edges. Cognitive, nonlinguistic tasks correlate with severity of the dementia, but tasks such as object naming, picture description and generative naming vary when severity is constant. Generative naming is, for still some uncharted reason, the most difficult for the Alzheimer patient and is also one of the most sensitive measures of that disease in its incipient stages.

Moving from dementia to aphasia, Martin and Dell trace the productions of anticipatory (right to left) movement errors and carryover/ perseverative (left to right) movement errors within the interactive spreading activation model developed by Dell. Again, this contribution demonstrates the unification of neuropsychology and connectionism, each in its own way emphasizing function. Perseveration now, of course, needs to make recourse to both horizontal (left to right) action as well as vertical action, whereby a prime is produced again in a later stimulus-response pair. Perseverations are things from the "past," as the title of Martin and Dell's article implies. "Past" can run in either a horizontal scheme or a vertical scheme. The "future," of course, represents the "look ahead" function of the serial ordering mechanism of Dell's model. That, too, can misfire, producing the anticipatory error. It turns out that perseverative errors are much more indicative of brain-damaged output, whereas anticipatory errors represent lessened pathological involvement. It has been long noted that corpuses of slips of the tongue from nonpathologically involved speakers comprise many more anticipatory movement errors, whereas movement errors in aphasia involve far more perseverative errors. Put another way, perseverative errors are indicative of brain damage; anticipatory errors are not. Martin and Dell use an "anticipatory proportion" ratio to measure severity of linear segmental paraphasias and use the same metric to chart recovery, whereby the AP (number of anticipations divided by number of anticipations + number of perseverations) increases, obviously as perseverations decrease. Their model simulations show the same sorts of relationships when "lesioned," in different ways by changing connection weights and decay rates. The AP, of course, may therefore be of great use as a metric to chart progress with such programs as the TAP. ${ }^{5}$

Focusing exclusively on perseverative errors, Buckingham and Christman analyze phonemic carryover errors as they form blended words. They show that the word initial syllable onset consonant or consonants are extremely vulnerable to perseveration and often serve as the intruding material that substitutes for target word onsets in the production of newly formed hybrid words. Similar to whole-word perseverations, these phonemic carryover phenomena are reflex like, automatic, and not under volitional control, although they may be subject to other linguistic conditions such as phoneme similarity, syllable slot sensitivity, the sonority principle, and phonotactics. It is argued as well that the nature of these kinds of errors helps garner support for certain phonological theories of syllable structure.

What does this all mean for individuals who perseverate? Anna Basso's article demonstrates, as does TAP, ${ }^{5}$ that the automaticity of perseveration represents a major control problem for the patient, which in turn represents a 
major challenge for the therapist. It also falls directly in line with the other contributions in this issue by stressing that the perseverative activity is not necessarily due to any abnormal increase in the inhibition of primes, but rather the perseveration involuntarily presents itself as a default behavior when the patient is required to respond in some way through a weakened (deafferented) system. Early on it was noted that perseverations occurred most often in optic aphasia, in which the disrupted system was visual object naming. It is now appreciated that practically any domain can be damaged, and if that damage also infiltrates some neuropharmacological system, then perseveration will likely be the default behavior. Basso diagnoses and treats the domain disorders of semantic appreciation and of writing, and notes that remediation in these areas will therefore reduce perseveration. Along the way, her therapy strategies and techniques force the clients' attention and focus on what they are doing and on the unwitting appearance of their perseverations. We mentioned earlier that the extremely rapid, reflex-like, cognitive processes involved with spreading activation, suppression, decay of nervous activity, and the like in normality are out of awareness and not under conscious or willful control. They represent activities that one cannot help but do. ${ }^{6}$ In pathology, this would still be the case, although the normal homeostasis is thrown out of balance. That is why patients are so often surprised or startled by their own perseverated elements, especially in the verbal domain. They often realize the errors ex post facto, but at least early on they have great difficulty controlling their realization. Although remediating the deafferented systems will in turn cut down on perseverations, part of the therapeutic intervention is also to help the patient catch and prevent them from occurring in the first place.

We want to thank our authors for their contributions and for their promptness, $\mathrm{pa}^{-}$ tience, and acceptance of our editorializing. We feel we have collected some of the most informed and prolific scholars investigating perseveration from a wide range of scientific approaches. We hope that all our readers, no matter what their clinical or research responsibilities may be, will carry away useful information about what we know, as well as about what we still do not know about perseveration. Fruitful inquiry always leaves much untapped. We also hope that the collectivity of the articles in this issue will serve to demonstrate the unification or alignment of very different levels of scientific description and explanation for the phenomena we call perseveration. Enjoy.

\section{REFERENCES}

1. Marie P. A singular trouble with speech: palilalia (dissociation of voluntary speech and of automatic speech). In: Cole MF, Cole M, eds. Pierre Marie's Papers on Speech Disorders. New York: Hafner; 1971:261-278. Originally published by Le Monde Medical 1925;664:329-344

2. Klein R, Albert M. Can drug therapies improve language functions of individuals with aphasia? A review of the evidence. Semin Speech Lang 2004; 25:193-204

3. Plaut DC, Shallice T. Perseverative and semantic influences on visual object naming errors in optic aphasia: a connectionist account. J Cog Neurosci 1993;5:89-117

4. Chomsky N. On Nature and Language. Cambridge, UK: Cambridge University Press; 2002

5. Helm-Estabrooks N, Albert M. Treatment for aphasic perseveration. In: Helm-Estabrooks N, Albert M, eds. Manual of Aphasia and Aphasia Therapy, 2nd ed. Austin, TX: Pro-Ed; 2004:201220

6. Fodor J. Precis of the modularity of mind. Behav Brain Sci 1985;8:1-41

Hugh W. Buckingham, Ph.D. Sarah S. Christman, Ph.D. Co-Guest Editors ${ }^{1,2}$ 